\title{
Contribuição dos diferentes alimentos segundo a classificação Nova para a ingestão de fibras alimentares em adolescentes
}

\author{
Contribution of different foods according to the Nova classification \\ to dietary fiber intake in adolescents
}

Rafaela de Campos Felippe Meira (https://orcid.org/0000-0002-8075-0447) ${ }^{1}$ Caroline Dario Capitani (https://orcid.org/0000-0002-3466-6148) ${ }^{2}$ Antonio de Azevedo Barros Filho (https://orcid.org/0000-0001-6239-1121) ${ }^{1}$ Marilisa Berti de Azevedo Barros (https://orcid.org/0000-0003-3974-195X) ${ }^{3}$ Daniela de Assumpção (https://orcid.org/0000-0003-1813-996X) ${ }^{1}$
${ }^{1}$ Departamento de Pediatria, Faculdade de Ciências Médicas, Universidade Estadual de Campinas (UNICAMP). R. Tessália Vieira de Camargo 126, Cidade Universitária. 13083-887 Campinas SP Brasil. danideassumpcao@ gmail.com

${ }^{2}$ Faculdade de Ciências Aplicadas, UNICAMP. Campinas SP Brasil. ${ }^{3}$ Departamento de Saúde Coletiva, Faculdade de Ciências Médicas, UNICAMP. Campinas SP Brasil.

\begin{abstract}
The aims of the present study were to evaluate dietary fiber intake and associated factors in adolescents, identify food sources of the nutrient, and determine associations between indicators of dietary patterns (energy/macronutrients/micronutrients) and dietary fiber intake. A population-based cross-sectional study was conducted involving 24-hour recall data. The NOVA classification was used to determine the contribution of foods to dietary fiber intake. Reference values from the World Health Organization $(\geq 12.5 \mathrm{~g})$ and the US Institute of Medicine (14 g) per 1,000 kcal were used to assess intake. The mean intake of dietary fiber $1,000 \mathrm{kcal} /$ day was $6.4 \mathrm{~g}(1.5 \mathrm{~g}$ of soluble fiber and $4.9 \mathrm{~g}$ of insoluble fiber) among the 891 adolescents. Fiber intake was low, especially among those who ate fruits, vegetables, and beans less, those who consumed soft drinks and processed meats more, and those who did not eat breakfast every day. Unprocessed/minimally processed foods provided 68.8\%, 53.7\%, and $72.1 \%$ of total, soluble, and insoluble fiber, respectively, whereas ultra-processed products provided $24.8 \%, 37.9 \%$, and $21.0 \%$ respectively. Fiber intake was inversely associated with energy intake, fat, free sugar, and animal protein in the diet. The insufficient fiber intake underscores the need for actions that promote healthy nutrition on the individual and family levels.
\end{abstract}

Key words Dietary Fiber, Adolescents, Health Surveys
Resumo Objetivou-se avaliar a ingestão de fibra alimentar e fatores associados em adolescentes; identificar as fontes alimentares do nutriente; $e$ verificar a relação de indicadores de práticas alimentares (energia/macro/micronutrientes) como consumo de fibras. Trata-se de estudo transversal de base populacional com dados de Recordatório de 24 Horas. Utilizou-se a classificação NOVA e foi avaliada a contribuição dos alimentos para o teor de fibras da dieta. Valores de referência da Organização Mundial da Saúde $(\geq 12,5 \mathrm{~g})$ e do Institute of Medicine $(14 \mathrm{~g})$ por $1.000 \mathrm{kcal}$ foram usados para avaliar o consumo. A ingestão média foi de $6,4 \mathrm{~g}$ de fibra alimentar $1.000 \mathrm{kcal} /$ dia, 1,5 $\mathrm{g}$ de solúvel e 4,9 $\mathrm{g}$ de insolúvel, para os 891 adolescentes. O consumo de fibras foi baixo, principalmente entre os que ingeriam menos frutas, hortaliças, feijão, mais refrigerante, embutidos, e nos que não consumiam o café da manhã diariamente. Os alimentos in natura forneceram $68,0 \%, 53,7 \%$ e $72,1 \%$ da fibra total, solúvel e insolúvel, e os ultraprocessados $24,8 \%, 37,9 \%$ e $21,0 \%$, respectivamente. O consumo de fibras foi inversamente associado ao teor de energia, gordura, açúcar livre e proteína animal da dieta. A ingestão insuficiente de fibras sinaliza a necessidade de promover a alimentação saudável e adequada em nível individual e familiar.

Palavras-chave Fibra Alimentar, Adolescentes, Inquéritos de Saúde 


\section{Introdução}

Estudos evidenciam que a maior ingestão de fibra alimentar diminui o risco de desenvolvimento de câncer de cólon proximal e distal ${ }^{1}$, de doenças cardiovasculares ${ }^{2,3}$ e de mortalidade por doenças cardíacas e neoplasias de todas as causas ${ }^{4}$. Pesquisa que analisou dados de três coortes prospectivas norte-americanas mostrou associação entre a qualidade do carboidrato e o risco de diabetes tipo 2; dietas com alto índice glicêmico ou alta carga glicêmica e menor quantidade de fibras de cereais aumentam, em torno de $50 \%$, o risco da doença ${ }^{5}$. Estudo com adolescentes europeus verificou uma relação inversa entre o consumo de fibra alimentar solúvel e níveis de glicose sanguínea $^{6}$. O inquérito de saúde e nutrição NHANES (2001-2012) identificou menor circunferência da cintura e Índice de Massa Corporal (IMC) nas crianças e adolescentes (6 a 18 anos) que mais ingeriam cereais integrais ${ }^{7}$.

Embora associado com benefícios à saúde, o consumo de fibra alimentar apresenta-se aquém dos valores recomendados. Segundo a Pesquisa de Orçamentos Familiares (POF, 2008-2009), a disponibilidade de fibra alimentar nos domicílios brasileiros era de 12,3 g/dia, e a recomendação de consumo da Organização Mundial da Saúde (OMS), adotada pelo Ministério da Saúde, é de no mínimo, 25,0 g/dia ${ }^{8}$. Nos Estados Unidos, a ingestão diária de fibra foi de 17,0 g nos adultos $\geq 19$ anos, e os valores de ingestão adequada variavam de 21 a $38 \mathrm{~g} /$ dia nessa população ${ }^{9}$. Na Austrália, a média do consumo de fibra foi de $23,8 \mathrm{~g} /$ dia e somente $28,2 \%$ dos adultos $(\geq 19$ anos) atingiram a recomendação diária de $30 \mathrm{~g}$ para homens e $25 \mathrm{~g}$ para mulheres ${ }^{10}$. Em adolescentes, a média de ingestão de fibra foi em torno de 20 g para brasileiros ${ }^{11}$, europeus ${ }^{6}$ e australianos ${ }^{10}$, e em torno de $13 \mathrm{~g}$ em crianças/adolescentes norte-americanos?.

Não existe uma definição universal para fibra alimentar, entretanto, uma das mais utilizadas foi proposta pelo Codex Alimentarius, que define o nutriente como polímeros de carboidratos com dez ou mais unidades monoméricas não hidrolisáveis por enzimas endógenas do intestino delgado de humanos ${ }^{12,13}$. O Codex considera como fibra alimentar os carboidratos encontrados naturalmente nos alimentos, e também os isolados ou extraídos da matriz alimentar e os sintetizados por processos industriais que apresentam benefícios à saúde comprovados cientificamente ${ }^{12}$.

A fibra alimentar é subdividida de acordo com as características de solubilidade em água, viscosidade e fermentação ${ }^{14-16}$. Fibras solúveis, viscosas, formam gel em contato com a água, condição que influencia a consistência do quimo, prolonga a digestão e absorção dos nutrientes, reduz o apetite, a absorção de colesterol e glicose. A fermentação (total ou parcial) da fibra produz ácidos graxos de cadeia curta, que fornecem energia para a mucosa do cólon, atuam na manutenção da integridade da barreira intestinal e regulação do sistema imune. As fibras insolúveis exercem efeito laxativo, aumentam o volume fecal e o tempo de trânsito intestinal ${ }^{14-16}$. As características da fibra alimentar coincidem nos alimentos, por isso a alimentação deve ser variada em frutas, hortaliças folhosas e tuberosas, feijões e cereais integrais.

A última edição do Guia Alimentar para a População Brasileira recomenda que os alimentos in natura ou minimamente processados, tais como frutas, hortaliças, raízes e tubérculos, grãos, leguminosas e oleaginosas sejam predominantes no repertório alimentar ${ }^{17}$. Esta recomendação torna-se fundamental diante do desafio contemporâneo de promover a ingestão adequada de fibra alimentar.

Considerando a importância da fibra alimentar para a saúde, a necessidade de avaliar o consumo de fibras e identificar os alimentos fontes na dieta, além da escassez de estudos de base populacional que analisaram o consumo do nutriente e suas frações, os objetivos do estudo foram: avaliar a ingestão de fibra alimentar e os fatores associados em adolescentes; identificar as fontes alimentares do nutriente; e verificar a relação de indicadores de práticas alimentares (energia/macro/micronutrientes) com o consumo de fibras.

\section{Métodos}

Neste estudo, foram utilizados dados de duas pesquisas de delineamento transversal, de base populacional, que envolveram pessoas não institucionalizadas e residentes na área urbana do município de Campinas-SP. A população de estudo foi constituída por três domínios de idade: adolescentes de 10 a 19 anos, adultos de 20 a 59 anos e idosos de 60 anos ou mais.

Uma das pesquisas, o Inquérito de Saúde de Campinas (ISACamp 2014-15) teve o objetivo de investigar características demográficas, socioeconômicas e múltiplas dimensões da saúde, tais como morbidades, uso de serviços de saúde, práticas preventivas, estilo de vida e consumo de medicamentos. O questionário foi preenchido 
em tablet por entrevistadores treinados, e estava organizado em 12 blocos temáticos.

O plano de amostragem do ISACamp 201415 baseou-se na obtenção de uma amostra probabilística, por conglomerados e em dois estágios: setor censitário e domicílio. No primeiro estágio, foi realizado sorteio aleatório de 70 setores censitários com probabilidade proporcional ao tamanho, dado pelo número de domicílios contados no Censo de 2010. Os setores foram ordenados pela renda média dos chefes do domicílio e, posteriormente, foi feita a seleção de 14 setores em cada um dos cinco distritos de saúde do município ${ }^{18}$.

Foi definido um tamanho mínimo de amostra para cada domínio de idade (1.000 para adolescentes e idosos, e 1.400 para adultos). Esses tamanhos de amostra foram estabelecidos considerando a situação correspondente à máxima variabilidade para a frequência dos eventos estudados $(\mathrm{p}=0,50)$, nível de confiança de $95 \%$ $(z=1,96)$, erro de amostragem entre $3 \%$ e $4 \%$ (adultos) e $4 \%$ e $5 \%$ (adolescentes e idosos), e efeito de delineamento de $2^{18}$.

No segundo estágio, foram definidos os números de domicílios necessários para atingir os tamanhos mínimos de amostra em cada domínio de idade. Deste modo, foram sorteados 3.119 domicílios para entrevistas com adolescentes, 1.029 para adultos e 3.161 para idosos, já prevendo taxas de não resposta de $27 \%, 22 \%$ e $20 \%$, respectivamente. Todos os moradores que pertenciam à faixa etária selecionada para aquele domicílio eram entrevistados ${ }^{18}$.

No presente estudo, também foram analisadas informações do Inquérito de Consumo Alimentar e Estado Nutricional (ISACamp-Nutri), um subprojeto de pesquisa desenvolvido na mesma amostra sorteada para o ISACamp. Depois de concluída a participação no ISACamp, entrevistadores retornavam ao domicílio para convidar os mesmos indivíduos a responderem um questionário de nutrição composto por Recordatório de 24 Horas (R24h), Questionário de Frequência Alimentar (QFA), perguntas sobre percepção corporal, práticas para a perda de peso, autoavaliação da qualidade da dieta, frequência de consumo de refeições, entre outras. A coleta dos dados foi padronizada por meio de treinamento, supervisão da equipe de campo e elaboração de materiais de apoio: manual do entrevistador, roteiro para a aplicação do R24h e manual fotográfico.

As entrevistas foram iniciadas pela aplicação do R24h, utilizando a abordagem "Por favor, me diga tudo o que comeu e bebeu ontem, desde o momento em que acordou até o horário em que foi dormir"19. O R24h foi conduzido por meio do Multiple-Pass Method, técnica que visa estimular a memória do respondente e obter estimativas mais precisas do consumo alimentar ${ }^{20}$. Baseiase numa entrevista estruturada em cinco passos: Quick list (relato espontâneo de todos os alimentos e bebidas ingeridos no dia anterior), Forgotten foods (checagem de alimentos frequentemente esquecidos), Time and eating occasion (registro do horário, nome e local de consumo das refeições), Detail Cycle (detalhamento de cada item alimentar, como as formas de preparo, a composição das preparações, o tipo e as respectivas quantidades), Final probe (revisão geral ${ }^{20}$.

Os alimentos e as preparações culinárias foram registrados em unidades ou medidas caseiras. Nutricionistas treinadas revisavam os R24h para identificar e corrigir possíveis falhas de preenchimento e, posteriormente, quantificavam os alimentos em unidades de peso ou volume. Para isto, foram utilizadas tabelas de medidas caseiras $^{21,22}$, rótulos de alimentos e serviços de atendimento ao consumidor. Os dados de consumo alimentar foram imputados no software Nutrition Data System for Research (NDS-R), versão 2015 (Nutrition Coordinating Center, Universidade de Minnesota) pela equipe de nutricionistas. As preparações culinárias típicas que não constavam na base de dados do programa foram elaboradas de acordo com receitas padronizadas ${ }^{21-23}$. Estas preparações ficam armazenadas no módulo de receitas do usuário (User Recipe), disponíveis para uso quando necessário.

O campo foi percorrido nos diferentes dias da semana, inclusive aos sábados e domingos, e as entrevistas tiveram duração média de $30 \mathrm{mi}-$ nutos (IC95\%: 28,6-31,3). O questionário do ISACamp-Nutri foi digitado em máscara desenvolvida com o uso do software EpiData versão 3.1 (EpiData Assoc., Odense, Dinamarca). Finalizada a entrada dos dados dietéticos no NDS-R e dos dados do questionário no EpiData, realizou-se análise de consistência para corrigir os erros de digitação.

\section{Variáveis utilizadas no estudo}

A variável de interesse foi a ingestão de fibra alimentar total, solúvel e insolúvel (g/dia) obtida do R24h. As quantidades de fibra alimentar total e das frações foram apresentadas em densidade energética $(\mathrm{g} / 1.000 \mathrm{kcal} / \mathrm{dia})$. Os valores de referência da OMS ( $\geq 12,5 \mathrm{~g} / 1.000 \mathrm{kcal})$ e do Institute 
of Medicine (IOM) (14 g/1.000 kcal) foram utilizados para avaliar a ingestão de fibra alimentar.

Foram consideradas como variáveis independentes:

Demográficas e socioeconômicas: sexo (masculino e feminino), faixa etária (10 a 14 e 15 a 19 anos), raça/cor da pele autorreferida (branca e não branca), escolaridade do chefe da família (0 a 4,5 a 8,9 a 11 e $\geq 12$ anos de estudo) e renda familiar per capita $(<0,5, \geq 0,5$ a $<1,0, \geq 1,0$ a $<1,5$ e $\geq 1,5$ salário mínimo).

Consumo alimentar, verificação do rótulo, autoavaliação da qualidade da dieta e Índice de Massa Corporal (IMC): frequência de consumo semanal de frutas, hortaliças cruas e cozidas, feijão, leite $(\geq 5 \text { e }<5 \text { vezes })^{24}$, refrigerante e embutidos $(\leq 2 \text { e }>2 \text { vezes })^{24}$, frequência semanal que realiza o café da manhã ( 7 vezes e $<7)^{24,25}$ e costume de verificar os rótulos de alimentos (não e sim/ às vezes ${ }^{26}$. A autoavaliação da qualidade da dieta foi obtida por meio da questão "Como você considera a qualidade da sua alimentação? Você acha que ela é...", categorizada em "muito boa/ boa", "regular", "ruim/muito ruim". Para o cálculo do IMC [peso $(\mathrm{kg}) /$ altura $\left.^{2}(\mathrm{~m})\right]$ foram utilizadas informações de peso referido e altura aferida, considerando que as medidas referidas de altura apresentam pior validade que as de peso, nos adolescentes ${ }^{27}$. O estado nutricional foi classificado de acordo com o IMC para idade em:magreza/eutrofia ( $\leq$ percentil 85$)$ e sobrepeso/obesidade $(>\text { percentil } 85)^{28}$.

Categoria de processamento dos alimentos: os 500 itens alimentares referidos no R24h foram codificados segundo a classificação NOVA, que considera a extensão e o propósito do processamento industrial dos alimentos ${ }^{29}$. Em seguida, os itens alimentares foram reunidos em quatro grupos: 1) alimentos in natura ou minimamente processados, composto por leguminosas, frutas, hortaliças folhosas e tuberosas, leite, carnes e ovos, cereais na forma de grãos e farinhas (arroz, aveia, trigo, milho, mandioca), oleaginosas, entre outros; 2) alimentos processados, incluindo pão francês, queijos, frutas em calda, hortaliças/ leguminosas conservadas em salmoura; 3) alimentos ultraprocessados, grupo compreendido por bebidas açucaradas, biscoitos, pães de forma, margarina, embutidos, achocolatado, macarrão instantâneo, guloseimas, salgadinhos de pacote; 4) ingredientes culinários, como manteiga, sal, açúcar e óleo ${ }^{29}$. A contribuição dos grupos e dos respectivos alimentos para o total de fibras foi apresentada em densidade energética e em percentual.
Indicadores de práticas alimentares: energia, carboidrato, proteína total, proteína de origem vegetal e animal, gordura total, gordura saturada e trans, açúcar de adição, colesterol, sódio e potássio. Os indicadores derivaram das informações do R24h e foram selecionados com base nas recomendações de consumo de nutrientes da $\mathrm{OMS}^{30,31}$.

\section{Análise de dados}

Foram calculadas as médias e o desvio padrão (DP) da densidade de fibra alimentar total, solúvel e insolúvel (g/1.000 kcal/dia), segundo as categorias das variáveis independentes. Também foram desenvolvidos modelos de regressão linear generalizado (GLM), ajustados por sexo, para estimar as médias da ingestão de fibra alimentar total e das frações solúvel e insolúvel. Em cada modelo foram incluídas as variáveis que apresentaram valor de $\mathrm{p}<0,20$ na análise bivariada e permaneceram aquelas com valor de $\mathrm{p}<0,05$. Técnicas gráficas e o Critério de Informação de Akaike (AIC) evidenciaram que a distribuição Gama se ajustava melhor aos dados de ingestão de fibras. Posteriormente, calculou-se a contribuição dos grupos alimentares e dos respectivos alimentos em relação ao conteúdo total de fibra alimentar e das frações. A análise de regressão linear foi empregada para verificar a relação dos indicadores de práticas alimentares com o consumo de fibras (categorias em quartis), e a significância geral do modelo foi determinada por meio do teste F. Foi adotado o nível de significância de 5\% para os testes estatísticos. Todas as análises foram realizadas no programa Stata versão 15.0, no módulo svy, que considera os pesos e o delineamento de amostragem.

\section{Procedimentos éticos}

O ISACamp e o ISACamp-Nutri foram aprovados pelo Comitê de Ética em Pesquisa da Universidade Estadual de Campinas e pela Comissão Nacional de Ética em Pesquisa (sistema CEP/CONEP). O projeto deste estudo foi aprovado pelo Comitê de Ética em Pesquisa da Unicamp.

\section{Resultados}

Dos 1.023 adolescentes entrevistados no ISACamp, 109 não participaram do ISACamp-Nutri (10,7\% de recusas ou perdas). Do total de 914 entrevistas, $866(94,7 \%)$ foram respondidas pelo 
próprio adolescente e outras $48(5,3 \%)$ pelos pais ou responsáveis, na maioria a mãe $(67,7 \%)$.

Quanto ao R24h, 11 indivíduos se recusaram a responder (adolescentes ou pais/responsáveis) e outros 12 recordatórios foram excluídos do presente estudo por apresentarem valor energético total considerado implausível $(<600 \mathrm{kcal}[\mathrm{n}=10]$ ou $>6.000 \mathrm{kcal}$ ). Portanto, neste estudo foram analisadas informações de 891 adolescentes, com média de idade de 14,6 anos (IC95\%: 14,4-14,8).

A amostra estudada apresentou proporções ligeiramente maiores de meninos $(52,0 \%)$, de adolescentes de 15 a 19 anos (52,7\%) e que se autodeclararam brancos (55,5\%). Quanto à escolaridade do chefe do domicílio, 19,7\% tinham até quatro anos de estudo e $26,1 \%$ das famílias recebiam menos que meio salário mínimo per capita. A baixa frequência de consumo de frutas ( $<5$ vezes/semana) foi referida por $61,9 \%$ dos adolescentes, de feijão ( $<5$ vezes/semana) por $28,3 \%$, enquanto elevada frequência de consumo de refrigerante ( $>2$ vezes/semana), por $50,7 \%$. O café da manhã foi consumido diariamente por $63,7 \%$ da amostra e $31,4 \%$ costumavam checar o rótulo dos alimentos (Tabelas 1 e 2).
A ingestão média total de fibras alimentares foi de 12,6 g (IC95\%: 12,2-13,2), da fração solúvel de 3,0 g (IC95\%: 2,8-3,2) e da fração insolúvel de 9,6 g (IC95\%: 9,2-10,0). Quanto ao sexo, as meninas apresentaram ingestão diária média de 11,7 g (IC95\%: 11,2-12,3), 2,9 g (IC95\%: 2,73,0) e $8,8 \mathrm{~g}$ (IC95\%: 8,4-9,3), e os meninos 13,5 g (IC95\%: 12,9-14,2), 3,1 g (IC95\%: 2,9-3,3) e 10,4 g (IC95\%: 9,8-10,9) de fibra alimentar total, solúvel e insolúvel, respectivamente (dados não apresentados em tabela).

O consumo diário de fibras alimentares foi estimado em 6,4 g/1.000 kcal para o conjunto dos adolescentes, valor correspondente a 1,5 g/1.000 kcal de fração solúvel e 4,9 g/1.000 kcal de fração insolúvel. As meninas tiveram maior ingestão de fibra alimentar total do que os meninos. O consumo de fibra alimentar solúvel mostrouse superior nas meninas e nos adolescentes que pertenciam aos estratos de maior escolaridade do chefe do domicílio ( $\geq 12$ em relação a $\leq 4$ anos de estudo) e renda familiar per capita ( $\geq 1,5$ em relação a $<0,5$ salário mínimo) (Tabela 1 ).

Na Tabela 2 observa-se que a ingestão de fibras alimentares foi inferior entre os adolescentes

Tabela 1. Médias de densidade de fibras alimentares e das frações solúvel e insolúvel segundo variáveis sociodemográficas, em adolescentes de 10 a 19 anos. ISACamp-Nutri 2014-2015.

\begin{tabular}{|c|c|c|c|c|c|c|c|}
\hline \multirow{2}{*}{ Variáveis } & \multirow{2}{*}{ n (\%) } & \multirow{2}{*}{$\begin{array}{c}\text { FA total } \\
\text { g/1.000 kcal }\end{array}$} & \multirow{2}{*}{ DP } & \multirow{2}{*}{$\begin{array}{c}\text { FA solúvel } \\
\text { g/1.000 kcal }\end{array}$} & \multirow{2}{*}{ DP } & \multirow{2}{*}{$\begin{array}{l}\text { FA insolúvel } \\
\text { g/1.000 kcal } \\
\end{array}$} & \multirow{2}{*}{ DP } \\
\hline & & & & & & & \\
\hline \multicolumn{8}{|l|}{ Sexo } \\
\hline Masculino & $463(52,1)$ & $6,2^{\mathrm{a}}$ & $\pm 2,4$ & $1,4^{\mathrm{a}}$ & $\pm 0,9$ & 4,8 & $\pm 2,1$ \\
\hline Feminino & $428(47,9)$ & $6,6^{\mathrm{b}}$ & $\pm 3,9$ & $1,6^{\mathrm{b}}$ & $\pm 1,4$ & 5,0 & $\pm 3,1$ \\
\hline Total & 891 & 6,4 & $\pm 2,7$ & 1,5 & $\pm 0,9$ & 4,9 & $\pm 2,4$ \\
\hline \multicolumn{8}{|c|}{ Faixa etária (em anos) } \\
\hline 10 a 14 & $422(47,4)$ & 6,4 & $\pm 2,5$ & 1,5 & $\pm 0,8$ & 4,8 & $\pm 2,0$ \\
\hline 15 a 19 & $469(52,6)$ & 6,5 & $\pm 3,5$ & 1,5 & $\pm 1,1$ & 4,8 & $\pm 2,8$ \\
\hline \multicolumn{8}{|l|}{ Raça/cor da pele } \\
\hline Branca & $487(55,5)$ & 6,4 & $\pm 2,9$ & 1,6 & $\pm 1,1$ & 4,8 & $\pm 2,4$ \\
\hline Não branca & $400(44,5)$ & 6,4 & $\pm 3,4$ & 1,5 & $\pm 1,4$ & 4,9 & $\pm 2,6$ \\
\hline \multicolumn{8}{|c|}{ Escolaridade do chefe da família (em anos) } \\
\hline 0 a 4 & $177(19,6)$ & 6,2 & $\pm 2,5$ & $1,4^{\mathrm{a}}$ & $\pm 1,1$ & 4,8 & $\pm 2,0$ \\
\hline 5 a 8 & $292(33,6)$ & 6,5 & $\pm 4,1$ & $1,6^{\mathrm{a}, \mathrm{b}}$ & $\pm 1,5$ & 5,0 & $\pm 3,0$ \\
\hline 9 a 11 & $257(28,9)$ & 6,1 & $\pm 3,5$ & $1,5^{\mathrm{a}, \mathrm{b}}$ & $\pm 1,6$ & 4,7 & $\pm 2,7$ \\
\hline 12 ou mais & $150(17,8)$ & 6,7 & $\pm 4,9$ & $1,8^{\mathrm{b}}$ & $\pm 1,6$ & 4,9 & $\pm 3,8$ \\
\hline \multicolumn{8}{|c|}{ Renda familiar per capita (em salário mínimo) } \\
\hline$<0,5$ & $234(26,1)$ & 6,4 & $\pm 2,6$ & $1,4^{\mathrm{a}}$ & $\pm 1,1$ & 5,0 & $\pm 2,4$ \\
\hline$\geq 0,5 \mathrm{a}<1,0$ & $302(33,0)$ & 6,5 & $\pm 4,2$ & $1,5^{\mathrm{a}, \mathrm{b}}$ & $\pm 1,4$ & 5,0 & $\pm 3,6$ \\
\hline$\geq 1,0 \mathrm{a}<1,5$ & $190(21,3)$ & 6,1 & $\pm 3,3$ & $1,5^{\mathrm{a}, \mathrm{b}}$ & $\pm 1,4$ & 4,6 & $\pm 2,7$ \\
\hline$\geq 1,5$ & $165(19,6)$ & 6,6 & $\pm 4,6$ & $1,7^{\mathrm{b}}$ & $\pm 1,5$ & 4,9 & $\pm 3,7$ \\
\hline
\end{tabular}

a,b: letras diferentes indicam diferenças estatisticamente significativas. 
Tabela 2. Médias de densidade de fibras alimentares e das frações solúvel e insolúvel segundo variáveis de consumo alimentar, verificação do rótulo, autopercepção da qualidade da dieta e Índice de Massa Corporal, em adolescentes de 10 a 19 anos. ISACamp-Nutri 2014-2015.

\begin{tabular}{|c|c|c|c|c|c|c|c|}
\hline \multirow{2}{*}{ Variáveis } & \multirow{2}{*}{$\mathbf{n}(\%)$} & \multirow{2}{*}{$\begin{array}{c}\text { FA total } \\
\mathrm{g} / 1.000 \mathrm{kcal}\end{array}$} & \multirow{2}{*}{ DP } & \multirow{2}{*}{$\begin{array}{c}\text { FA solúvel } \\
\text { g/1.000 kcal }\end{array}$} & \multirow{2}{*}{ DP } & \multirow{2}{*}{$\begin{array}{c}\text { FA insolúvel } \\
\text { g/1.000 kcal }\end{array}$} & \multirow{2}{*}{ DP } \\
\hline & & & & & & & \\
\hline \multicolumn{8}{|l|}{ Consumo de frutas } \\
\hline$\geq 5$ vezes na semana & $330(38,0)$ & $7,1^{\mathrm{a}}$ & $\pm 3,2$ & $1,7^{\mathrm{a}}$ & $\pm 1,1$ & $5,4^{\mathrm{a}}$ & $\pm 2,5$ \\
\hline$<5$ vezes na semana & $561(62,0)$ & $6,0^{\mathrm{b}}$ & $\pm 4,7$ & $1,4^{\mathrm{b}}$ & $\pm 1,6$ & $4,5^{\mathrm{b}}$ & $\pm 3,5$ \\
\hline \multicolumn{8}{|c|}{ Consumo de hortaliças cruas } \\
\hline$\geq 5$ vezes na semana & $246(28,3)$ & $7,1^{\mathrm{a}}$ & $\pm 3,1$ & $1,7^{\mathrm{a}}$ & $\pm 1,1$ & $5,4^{\mathrm{a}}$ & $\pm 2,6$ \\
\hline$<5$ vezes na semana & $645(71,7)$ & $6,1^{\mathrm{b}}$ & $\pm 5,3$ & $1,5^{\mathrm{b}}$ & $\pm 2,0$ & $4,7^{\mathrm{b}}$ & $\pm 4,3$ \\
\hline \multicolumn{8}{|c|}{ Consumo de hortaliças cozidas } \\
\hline$\geq 5$ vezes na semana & $99(11,2)$ & $7,4^{\mathrm{a}}$ & $\pm 4,1$ & $1,8^{\mathrm{a}}$ & $\pm 1,6$ & $5,5^{\mathrm{a}}$ & $\pm 3,0$ \\
\hline$<5$ vezes na semana & $792(88,8)$ & $6,2^{\mathrm{b}}$ & $\pm 11,8$ & $1,4^{\mathrm{b}}$ & $\pm 4,5$ & $4,7^{\mathrm{b}}$ & $\pm 8,4$ \\
\hline \multicolumn{8}{|l|}{ Consumo de leite } \\
\hline$\geq 5$ vezes na semana & $469(52,9)$ & 6,5 & $\pm 2,6$ & 1,5 & $\pm 1,1$ & 4,9 & $\pm 2,2$ \\
\hline$<5$ vezes na semana & $422(47,1)$ & 6,3 & $\pm 3,3$ & 1,5 & $\pm 1,4$ & 4,7 & $\pm 2,5$ \\
\hline \multicolumn{8}{|l|}{ Consumo de feijão } \\
\hline$\geq 5$ vezes na semana & $641(71,7)$ & $6,6^{\mathrm{a}}$ & $\pm 2,3$ & $1,5^{\mathrm{a}}$ & $\pm 1,0$ & $5,1^{\mathrm{a}}$ & $\pm 2,3$ \\
\hline$<5$ vezes na semana & $250(28,3)$ & $6,0^{\mathrm{b}}$ & $\pm 3,6$ & $1,7^{\mathrm{b}}$ & $\pm 1,4$ & $4,2^{\mathrm{b}}$ & $\pm 2,7$ \\
\hline \multicolumn{8}{|l|}{ Consumo de refrigerante } \\
\hline$\leq 2$ vezes na semana & $441(49,4)$ & $6,7^{\mathrm{a}}$ & $\pm 2,7$ & $1,6^{\mathrm{a}}$ & $\pm 1,0$ & $5,1^{\mathrm{a}}$ & $\pm 2,3$ \\
\hline$>2$ vezes na semana & $450(50,6)$ & $6,0^{\mathrm{b}}$ & $\pm 3,4$ & $1,4^{\mathrm{b}}$ & $\pm 1,3$ & $4,6^{\mathrm{b}}$ & $\pm 2,7$ \\
\hline \multicolumn{8}{|l|}{ Consumo de embutidos } \\
\hline$\leq 2$ vezes na semana & $495(55,4)$ & $6,6^{\mathrm{a}}$ & $\pm 3,1$ & 1,6 & $\pm 1,1$ & $5,0^{\mathrm{a}}$ & $\pm 2,4$ \\
\hline$>2$ vezes na semana & $396(44,6)$ & $6,2^{\mathrm{b}}$ & $\pm 3,6$ & 1,5 & $\pm 1,4$ & $4,7^{\mathrm{b}}$ & $\pm 2,8$ \\
\hline \multicolumn{8}{|l|}{ Café da manhã (frequência) } \\
\hline 7 vezes na semana & $569(63,8)$ & $6,7^{\mathrm{a}}$ & $\pm 2,6$ & $1,6^{\mathrm{a}}$ & $\pm 0,9$ & $5,0^{\mathrm{a}}$ & $\pm 2,1$ \\
\hline$<7$ vezes na semana & $322(36,2)$ & $5,9^{\mathrm{b}}$ & $\pm 3,4$ & $1,4^{\mathrm{b}}$ & $\pm 1,1$ & $4,5^{\mathrm{b}}$ & $\pm 2,7$ \\
\hline \multicolumn{8}{|c|}{ Verifica os rótulos dos alimentos } \\
\hline Não & $613(68,7)$ & $6,2^{\mathrm{a}}$ & $\pm 2,5$ & $1,5^{\mathrm{a}}$ & $\pm 1,0$ & $4,7^{\mathrm{a}}$ & $\pm 2,2$ \\
\hline Sim/às vezes & $278(31,3)$ & $6,8^{\mathrm{b}}$ & $\pm 3,7$ & $1,7^{\mathrm{b}}$ & $\pm 1,3$ & $5,1^{\mathrm{b}}$ & $\pm 2,8$ \\
\hline \multicolumn{8}{|c|}{ Percepção da qualidade da dieta } \\
\hline Muito boa/boa & $509(57,3)$ & $6,5^{\mathrm{a}}$ & $\pm 2,7$ & 1,5 & $\pm 1,1$ & $5,0^{\mathrm{a}}$ & $\pm 2,3$ \\
\hline Regular & $311(34,6)$ & $6,4^{\mathrm{a}, \mathrm{b}}$ & $\pm 2,6$ & 1,5 & $\pm 1,0$ & $4,9^{\mathrm{a}, \mathrm{b}}$ & $\pm 2,1$ \\
\hline Ruim/muito ruim & $71(8,1)$ & $5,6^{\mathrm{b}}$ & $\pm 3,2$ & 1,6 & $\pm 1,5$ & $4,0^{\mathrm{b}}$ & $\pm 2,1$ \\
\hline \multicolumn{8}{|l|}{$\operatorname{IMC}\left(\mathrm{kg} / \mathrm{m}^{2}\right)$} \\
\hline Baixo peso/eutrofia & $543(66,5)$ & 6,4 & $\pm 2,7$ & 1,6 & $\pm 0,9$ & 4,8 & $\pm 2,1$ \\
\hline Sobrepeso/obesidade & $275(33,5)$ & 6,5 & $\pm 2,8$ & 1,6 & $\pm 1,2$ & 4,9 & $\pm 2,3$ \\
\hline
\end{tabular}

n: número de adolescentes na amostra não ponderada; \%: Porcentagem na amostra ponderada; FA: fibra alimentar; DP: desvio padrão; ${ }^{\text {a,b }}$ : letras diferentes indicam diferenças estatisticamente significativas; IMC: Índice de Massa Corporal.

Fonte: Inquérito de Consumo Alimentar e Estado Nutricional do município de Campinas-SP, Brasil (ISACamp-Nutri, 2014-2015).

que consumiam frutas, hortaliças cruas, cozidas e feijão com frequência semanal menor que cinco vezes, assim como refrigerantes e embutidos mais que duas vezes na semana. Aqueles que não consumiam o café da manhã diariamente e os que não costumavam verificar o rótulo dos alimentos apresentaram médias inferiores de ingestão de fibras alimentares. Verificou-se menor ingestão de fibra alimentar total e insolúvel entre os que autoavaliaram a qualidade da dieta como ruim/ muito ruim, comparados aos que responderam muito boa ou boa.

A Tabela 3 apresenta os resultados dos modelos de regressão linear generalizado para fibra alimentar total, solúvel e insolúvel. Os segmentos de adolescentes que consumiam frutas, hortaliças cruas e feijão com menor frequência semanal, refrigerantes e embutidos com maior frequência, 
Tabela 3. Modelos de regressão linear generalizado. Variáveis associadas à densidade de fibra alimentar (g/1.000 kcal) total, solúvel e insolúvel. ISACamp-Nutri 2014-2015.

\begin{tabular}{|c|c|c|}
\hline \multirow{2}{*}{ Variáveis } & \multicolumn{2}{|c|}{ Modelo 1 - Fibra alimentar total ${ }^{\star}$} \\
\hline & Estimativa & Valor de p \\
\hline Intercepto & 7,9 & \\
\hline Sexo (feminino)/(masculino) & 0,36 & 0,064 \\
\hline Frutas $(<5$ dias semana $) /(\geq 5)$ & $-0,78$ & $<0,001$ \\
\hline Hortaliças cruas $(<5$ dias semana $) /(\geq 5)$ & $-0,60$ & 0,001 \\
\hline Feijão $(<5$ dias semana $) /(\geq 5)$ & $-0,55$ & 0,007 \\
\hline Refrigerante ( $>2$ dias semana) $/(\leq 2)$ & $-0,32$ & 0,037 \\
\hline Embutidos $(>2$ dias semana $) /(\leq 2)$ & $-0,40$ & 0,014 \\
\hline Café da manhã ( $<7$ dias semana)/(7) & $-0,60$ & 0,001 \\
\hline \multirow{2}{*}{ Variáveis } & \multicolumn{2}{|c|}{ Modelo 2 - Fibra alimentar solúvel ${ }^{\star *}$} \\
\hline & Estimativa & Valor de p \\
\hline Intercepto & 1,7 & \\
\hline Sexo (feminino)/(masculino) & 0,14 & 0,035 \\
\hline Escolaridade $(12$ anos ou +$) /(0$ a 4$)$ & 0,26 & 0,036 \\
\hline Frutas $(<5$ dias semana $) /(\geq 5)$ & $-0,14$ & 0,024 \\
\hline Hortaliças cruas $(<5$ dias semana $) /(\geq 5)$ & $-0,17$ & 0,010 \\
\hline Feijão $(<5$ dias semana $) /(\geq 5)$ & 0,27 & 0,002 \\
\hline Refrigerante ( $>2$ dias semana) $/(\leq 2)$ & $-0,16$ & 0,013 \\
\hline Café da manhã ( $<7$ dias semana)/(7) & $-0,23$ & $<0,001$ \\
\hline \multirow{2}{*}{ Variáveis } & \multicolumn{2}{|c|}{ Modelo 3 - Fibra alimentar insolúvel ${ }^{\star * *}$} \\
\hline & Estimativa & Valor de p \\
\hline Intercepto & 6,1 & \\
\hline Sexo (feminino)/(masculino) & 0,22 & 0,143 \\
\hline Frutas $(<5$ dias semana $) /(\geq 5)$ & $-0,66$ & $<0,001$ \\
\hline Hortaliças cruas $(<5$ dias semana $) /(\geq 5)$ & $-0,47$ & 0,004 \\
\hline Feijão $(<5$ dias semana $) /(\geq 5)$ & $-0,82$ & $<0,001$ \\
\hline Embutidos $(>2$ dias semana $) /(\leq 2)$ & $-0,36$ & 0,008 \\
\hline Café da manhã ( $<7$ dias semana)/(7) & $-0,44$ & 0,004 \\
\hline
\end{tabular}

*Variáveis inseridas no modelo 1: sexo, frequência de consumo semanal de frutas, feijão, hortaliças cruas, cozidas, refrigerante e embutidos, autoavaliação da qualidade da dieta, costume de verificar os rótulos de alimentos e frequência semanal de consumo do café da manhã; ${ }^{*}$ Variáveis inseridas no modelo 2: sexo, raça/cor da pele, escolaridade do chefe de família, renda familiar, consumo semanal de frutas, feijão, hortaliças cruas, cozidas e refrigerante, costume de verificar os rótulos de alimentos e frequência semanal de consumo do café da manhã; ${ }^{* * *}$ Variáveis introduzidas no modelo 3: sexo, consumo semanal de frutas, feijão, hortaliças cruas, cozidas, leite, refrigerante e embutidos, autoavaliação da qualidade da dieta, costume de verificar os rótulos de alimentos e frequência semanal de consumo do café da manhã.

Fonte: Inquérito de Consumo Alimentar e Estado Nutricional do município de Campinas-SP, Brasil (ISACamp-Nutri, 2014-2015).

e os que não consumiam o café da manhã diariamente tiveram médias mais baixas de fibra alimentar total. As médias de fibra solúvel foram superiores nas meninas e nos adolescentes cujos chefes de família eram mais escolarizados, e inferiores nos segmentos que consumiam frutas, hortaliças cruas e feijão com menor frequência semanal, refrigerante mais do que duas vezes na semana e nos que não consumiam o café da manhã diariamente. A menor ingestão de fibra alimentar insolúvel esteve associada à menor frequência de consumo semanal de frutas, hortaliças cruas, feijão ( $<5$ dias), embutidos ( $>2$ dias) e café da manhã ( $<7$ dias).

O grupo de alimentos in natura ou minimamente processados forneceu $68,0 \%$ da fibra alimentar total diária, o de alimentos processados $7,2 \%$ e o de ultraprocessados $24,8 \%$. No grupo dos alimentos in natura, o feijão, os cereais, as frutas e as raízes e tubérculos representaram as principais fontes de fibras alimentares, destacando-se a contribuição do feijão para o total de fibra insolúvel. Entre os alimentos processados, o pão francês foi o que mais contribuiu com fibras, 
já entre os ultraprocessados foram os biscoitos, os pães de forma e o achocolatado. Em relação ao total de fibra solúvel, aproximadamente $38,0 \%$ foi proveniente de alimentos ultraprocessados (Tabela 4).

$\mathrm{O}$ aumento da ingestão de fibras alimentares esteve associado à redução do conteúdo de energia, proteína de origem animal, gordura total, gordura saturada, colesterol e açúcares de adição da dieta, e associado ao aumento do conteúdo de carboidratos, proteína de origem vegetal e potássio (Tabela 5).

\section{Discussão}

Neste estudo, a ingestão média de fibra alimentar ajustada por energia foi de $6,4 \mathrm{~g} / 1.000 \mathrm{kcal} / \mathrm{dia} \mathrm{e}$ a total foi de $12,7 \mathrm{~g}$ (13,5 g nos meninos e 11,7 $\mathrm{g}$ nas meninas). Esses valores ficaram aquém das recomendações propostas pela OMS ( $\geq 25 \mathrm{~g} /$ dia ou $12,5 \mathrm{~g} / 1.000 \mathrm{kcal})^{30}$ e pelo IOM (38 g/dia para meninos e $26 \mathrm{~g}$ para meninas ou $14 \mathrm{~g} / 1.000 \mathrm{kcal} /$ dia) $)^{32}$.

A ingestão de fibra alimentar observada nesta pesquisa foi inferior à encontrada em adolescentes da Europa $\left(20,3\right.$ g e 8,4 g/1.000 kcal/dia) ${ }^{6}$, dos Estados Unidos (13,2 g/dia $)^{9}$ e da Austrália
$(19,8 \mathrm{~g} /$ dia para 9 a 13 anos e $18,8 \mathrm{~g} /$ dia para 14 a 18 anos $)^{10}$. Foi também inferior ao consumo de fibras estimado em adolescentes brasileiros, que foi de $20,4 \mathrm{~g}$ ( $10 \mathrm{a} 13$ anos) e 23,4 g (14 a 18 anos) no sexo masculino e em $18,8 \mathrm{~g}$ e $18,5 \mathrm{~g}$ no sexo feminino, respectivamente ${ }^{11}$.

O padrão alimentar da população brasileira sofreu intensas transformações nas últimas décadas, decorrentes da redução do consumo de alimentos tradicionais como arroz, feijão e mandioca, e aumento do consumo de produtos ultraprocessados como biscoitos, sorvetes e lanches do tipo fast-food ${ }^{33,34}$. Além da inerente escassez de fibras e outros nutrientes, esses produtos possuem características que estimulam o consumo excessivo: são extremamente saborosos, práticos, amplamente propagandeados e comercializados, e acessíveis a todos os estratos sociais da população ${ }^{17}$. No Brasil, dados da POF 2008-2009 mostravam que $69,5 \%$ e $21,5 \%$ da ingestão total de energia eram provenientes de alimentos in natura e de produtos ultraprocessados ${ }^{35}$, o que no Reino Unido correspondia a $28,6 \%$ e $50,7 \%$ para o ano de $2008^{36}$.

Na perspectiva do processamento de alimentos, as fibras dietéticas são adicionadas em produtos alimentícios porque desempenham funções tecnológicas como a capacidade de retenção

Tabela 4. Densidade e contribuição dos grupos de alimentos/itens alimentares em relação aos totais (em gramas) de fibra alimentar e das frações solúvel e insolúvel. ISACamp-Nutri 2014-2015.

\begin{tabular}{lrrrrrr}
\hline \multirow{2}{*}{ Grupos de alimentos } & \multicolumn{3}{c}{ Densidade (g/1.000 kcal/dia) } & \multicolumn{3}{c}{ Contribuição (\%) } \\
\cline { 2 - 7 } & FAT & FAS & FAI & FAT & \multicolumn{1}{c}{ FAS } & FAI \\
\hline In natura/minimamente processados & 4,2 & 0,8 & 3,4 & 68,0 & 53,7 & 72,1 \\
Feijão & 1,7 & 0,1 & 1,6 & 28,2 & 4,7 & 35,1 \\
Cereais & 0,7 & 0,2 & 0,5 & 10,8 & 15,5 & 9,5 \\
Frutas & 0,6 & 0,2 & 0,4 & 9,1 & 15,1 & 7,4 \\
Raízes e tubérculos & 0,4 & 0,2 & 0,2 & 7,1 & 13,6 & 5,2 \\
Arroz & 0,4 & 0,0 & 0,4 & 6,3 & 0,2 & 8,1 \\
Hortaliças & 0,4 & 0,1 & 0,3 & 5,9 & 4,6 & 6,3 \\
Processados & 0,4 & 0,1 & 0,3 & 7,2 & 8,4 & 6,9 \\
Pão francês & 0,4 & 0,1 & 0,3 & 6,5 & 7,6 & 6,2 \\
Ultraprocessados & 1,5 & 0,5 & 1,0 & 24,8 & 37,9 & 21,0 \\
Biscoitos & 0,3 & 0,1 & 0,2 & 5,2 & 8,8 & 4,2 \\
Pães & 0,3 & 0,1 & 0,2 & 4,5 & 8,1 & 3,5 \\
Achocolatado & 0,2 & 0,0 & 0,2 & 3,5 & 3,3 & 3,6 \\
Molhos industrializados & 0,2 & 0,1 & 0,1 & 2,9 & 3,7 & 2,8 \\
Doces & 0,2 & 0,1 & 0,1 & 2,8 & 4,2 & 2,3 \\
Salgadinho & 0,1 & 0,0 & 0,1 & 2,2 & 2,5 & 2,1 \\
Macarrão instantâneo & 0,1 & 0,0 & 0,1 & 1,4 & 1,8 & 1,3 \\
\hline
\end{tabular}

FAT: fibra alimentar total; FAS: fibra alimentar solúvel; FAI: fibra alimentar insolúvel. 
Tabela 5. Média de ingestão de energia e nutrientes segundo a densidade de fibras alimentares em quartil, em adolescentes de 10 a 19 anos. ISACamp-Nutri 2014-2015.

\begin{tabular}{|c|c|c|c|c|c|c|}
\hline \multirow{2}{*}{ Variáveis } & \multirow{2}{*}{ Média Total } & \multicolumn{4}{|c|}{ Densidade de FA (g/1.000 kcal) em quartil } & \multirow{2}{*}{$\begin{array}{c}\text { Valor de } \\
\mathbf{p}^{*}\end{array}$} \\
\hline & & 1 & 2 & 3 & 4 & \\
\hline Energia (kcal/dia) & 2034,0 & 2164,8 & 2142,2 & 1978,8 & 1849,5 & $<0,001$ \\
\hline \multicolumn{7}{|c|}{$\%$ de energia proveniente de: } \\
\hline Carboidratos & 50,6 & 46,5 & 49,6 & 51,8 & 54,8 & $<0,001$ \\
\hline Proteína total & 15,8 & 16,6 & 15,7 & 15,5 & 15,4 & 0,083 \\
\hline Proteína vegetal & 5,4 & 4,0 & 5,2 & 5,9 & 6,6 & $<0,001$ \\
\hline Proteína animal & 10,4 & 12,6 & 10,6 & 9,6 & 8,8 & $<0,001$ \\
\hline Gordura total & 33,6 & 36,6 & 34,6 & 32,7 & 30,4 & $<0,001$ \\
\hline Gordura saturada & 10,8 & 12,4 & 11,4 & 10,3 & 9,2 & $<0,001$ \\
\hline Gordura trans & 1,2 & 1,4 & 1,2 & 1,2 & 1,1 & 0,064 \\
\hline Açúcar livre & 11,8 & 15,0 & 12,3 & 10,2 & 9,4 & $<0,001$ \\
\hline Colesterol (mg) & 239,2 & 311,2 & 244,8 & 212,4 & 187,7 & $<0,001$ \\
\hline Sódio $(\mathrm{g})$ & 3,5 & 3,5 & 3,7 & 3,4 & 3,3 & 0,072 \\
\hline Potássio (mg) & 1968,8 & 1724,0 & 1953,5 & 1996,5 & 2203,1 & $<0,001$ \\
\hline
\end{tabular}

FA: fibra alimentar; Intervalo de FA (g/1.000 kcal) em cada quartil: $1(0,46 \mathrm{a} \leq 4,78), 2(>4,78 \mathrm{a} \leq 6,20), 3(>6,20 \mathrm{a} \leq 7,64), 4(>7,64 \mathrm{a}$ $22,58) ;{ }^{*}$ Teste $\mathrm{F}$ : utilizado para verificar a significância do modelo de regressão linear.

Fonte: Inquérito de Consumo Alimentar e Estado Nutricional do município de Campinas-SP, Brasil (ISACamp-Nutri, 2014-2015).

de água e gordura, espessamento, modificação de textura e sabor, formação de gel, controle da cristalização do açúcar, entre outras ${ }^{37}$. A inulina, por exemplo, é uma fibra solúvel utilizada em substituição à gordura nos produtos de panificação, laticínios, molhos e pastas cremosas para pães ${ }^{37}$. Nos Estados Unidos há uma diversidade de carboidratos não digeríveis sintéticos (polidextrose, fruto-oligossacarídeos de cadeia curta, galacto-oligosacarídeos) e isolados (alginato, fibras da parede celular de plantas) para uso comercial, que foram definidos como fibras dietéticas pela Food and Drug Administration ${ }^{38}$. É importante ressaltar que independentemente da presença desses tipos de fibras, os produtos ultraprocessados não são saudáveis.

$\mathrm{Na}$ análise bivariada, o sexo feminino apresentou maior consumo de fibra alimentar total e solúvel, em comparação ao masculino. Já no modelo final, observou-se maior ingestão de fibra solúvel nas meninas. Entre os participantes do estudo transversal HELENA-CCS (Healthy Lifestyle in Europe by Nutrition in Adolescence), a ingestão de fibras alimentares foi superior nas meninas $(8,9 \mathrm{~g} / 1.000 \mathrm{kcal}$ versus $7,8 \mathrm{~g} / 1.000 \mathrm{kcal}$; $\mathrm{p}<0,001)$, assim como o atendimento à recomendação do IOM $(11,4 \% \text { versus } 2,1 \%)^{6}$. Na Austrália, não foram detectadas diferenças por sexo na proporção de adolescentes que atingiram os valores de referência estabelecidos para a população do País ${ }^{10}$. Entre os adolescentes brasileiros, a prevalência de inadequação do consumo de fibras alimentares $(\leq 12,5 \mathrm{~g} / 1.000 \mathrm{kcal})$ foi maior nas meninas, alcançando $86,0 \%$ no grupo de 14 a 18 anos $^{11}$. Resultados de inquéritos nacionais mostram que a adesão ao padrão alimentar não saudável é maior nos adolescentes do sexo feminino, comparado ao masculino ${ }^{24,39}$. Maia et al. ${ }^{24}$ observaram que o padrão alimentar não saudável, caracterizado pelo consumo de doces, salgados fritos/batata frita, macarrão instantâneo, salgadinhos de pacote, biscoitos salgados e embutidos, associou-se com outros comportamentos inadequados, como o hábito de não consumir o café da manhã, de não realizar as refeições com os pais e de frequentar restaurantes fast-food. Portanto, o achado deste estudo pode decorrer da maior ingestão de alimentos não saudáveis entre as meninas.

O consumo de fibra solúvel foi maior nos domicílios em que os chefes de família eram mais escolarizados, em relação àqueles com até quatro anos de estudo. Pesquisa conduzida na Austrália evidenciou que a ingestão de fibras alimentares era superior no segmento de maior renda, e que $33,3 \%$ dos mais pobres e $51,1 \%$ dos mais ricos alcançaram os níveis recomendados do nutriente ${ }^{10}$. Em adolescentes brasileiros cujas mães possuem ensino superior completo, observou-se maior frequência de consumo de frutas e hortaliças, em relação às mães com ensino fundamental incompleto $^{40}$. O rápido crescimento da participação de 
produtos prontos ou quase prontos para o consumo e a redução de alimentos in natura na dieta tem atravessado todos os segmentos sociais da população $0^{34}$. Dados da POF 2008-2009 mostraram queda de 7,5\% na participação calórica de alimentos in natura e crescimento de 19,3\% na participação de produtos prontos entre a população classificada no primeiro e no último quintil de renda ${ }^{34}$.

Verificou-se menor ingestão de fibras alimentares entre os adolescentes que consumiam menos frutas, hortaliças cruas e feijão, e mais embutidos e refrigerantes durante a semana. Os alimentos de origem vegetal apresentam quantidades variáveis de fibra alimentar e as principais fontes provêm de cereais, preferencialmente integrais, tubérculos, leguminosas, hortaliças e frutas ${ }^{14-16}$. A pectina, uma fibra solúvel, viscosa e fermentável, é encontrada nas frutas, leguminosas, aveia, batata e no albedo da laranja (parte branca sob a casca), já a celulose, uma fibra insolúvel, não fermentável, está presente em cereais, hortaliças, cascas de frutas, castanhas e sementes $^{14-16}$. Dados da POF 2008-2009 demonstram que o consumo de ultraprocessados afeta negativamente a qualidade da alimentação, sendo que $o$ teor de fibras é quase três vezes menor na fração da dieta referente aos ultraprocessados, em relação a de alimentos in natura ${ }^{35}$.

Neste estudo, também foi verificada menor ingestão de fibra alimentar total, solúvel e insolúvel entre os indivíduos que não consumiam o café da manhã diariamente. Resultados do Estudo de Riscos Cardiovasculares em Adolescentes de 1217 anos (ERICA 2013-2014) apontam que 21,9\% nunca consumiam o café da manhã $(25,8 \%$ das meninas e $18,2 \%$ dos meninos) ${ }^{41}$. Em São Paulo, o não consumo do café da manhã alcançou 38,0\%, enquanto que a contribuição desta refeição para o total de fibras na dieta dos adolescentes foi de quase $20,0 \%$ (4,0 g para os meninos e $3,4 \mathrm{~g}$ para as meninas $)^{42}$. No Reino Unido, crianças e adolescentes ( 4 a 18 anos) que consumiam o café da manhã todos os dias apresentaram maior ingestão de fibras alimentares, cálcio, ferro e folato ${ }^{43}$. O café da manhã constitui uma das três principais refeições do $\mathrm{dia}^{17}$, e seu consumo é importante para aumentar o aporte de fibras alimentares com a oferta de frutas, pães e cereais integrais.

$\mathrm{Na}$ análise bivariada, verificou-se associação da autoavaliação da qualidade da dieta como ruim e a menor ingestão de fibras e do costume de checar os rótulos dos alimentos com o maior consumo do nutriente. Estes achados revelam a importância dos hábitos alimentares e dos com- portamentos saudáveis para promover o consumo de alimentos fontes de fibras. Um estudo identificou pior qualidade global da dieta, menor ingestão de cereais integrais, frutas e maior de gorduras sólidas e açúcar de adição entre os adolescentes que consideravam a alimentação de qualidade ruim/muito ruim $^{44}$.

Em Campinas-SP, 31,2\% (IC95\%: 26,8-35,9) dos adolescentes costumavam checar o rótulo dos alimentos, os quais verificavam, principalmente, a data de validade $(82,1 \%)$, as calorias $(19,7 \%)$, o teor de gordura $(10,7 \%)$ e, apenas $1,0 \%$, checava o teor de fibras alimentares. Portanto, a associação entre a ingestão de fibras e a leitura de rótulos não reflete a preocupação do adolescente sobre o conteúdo do nutriente, mas provavelmente por outras características do alimento.

Nesta pesquisa, os alimentos in natura/minimamente processados forneceram $68,0 \%$ do conteúdo total de fibras alimentares, $53,7 \%$ da fração solúvel e $72,1 \%$ da insolúvel. No conjunto da população brasileira ( $\geq 10$ anos), os alimentos que mais contribuíram com fibras alimentares foram: feijão $(36,9 \%)$, arroz $(9,8 \%)$, pães $(9,3 \%)$, hortaliças $(7,8 \%)$, frutas $(7,7 \%)$ e farinha de mandioca $(5,5 \%)^{8}$. Em adolescentes europeus, os alimentos que mais forneceram fibras foram os pães (20,8\%, $24,4 \%$ e $18,4 \%$ das fibras totais, solúveis e insolúveis, respectivamente), cereais, raízes e tubérculos $(17,5 \%, 20,1 \%, 17,9 \%)$, doces/salgadinhos $(16,7 \%, 11,5 \%, 19,1 \%)$, frutas $(13,9 \%$, $13,1 \%, 14,0 \%)$ e hortaliças $(9,8 \%, 9,3 \%, 10,0 \%)^{6}$. Os produtos ultraprocessados forneceram quase $25,0 \%$ do total de fibras da dieta dos adolescentes de Campinas. Este resultado demanda reflexão, pois a contribuição desses produtos para o teor de fibras equivale a sua contribuição energética, ou seja, na medida em que aumentar o consumo de ultraprocessados, a tendência será de redução na ingestão de fibras alimentares ${ }^{35}$. Como já citado, os alimentos ultraprocessados são nutricionalmente desbalanceados e devem ser evitados de acordo com a recomendação do Guia Alimentar para a População Brasileira ${ }^{17}$.

$\mathrm{O}$ aumento da ingestão de fibras foi inversamente associado ao teor de energia, proteína animal, gordura total, gordura saturada, colesterol e açúcar livre da alimentação. As fibras dietéticas são naturalmente encontradas em alimentos de origem vegetal, os quais são boas fontes de nutrientes e costumam apresentar menor densidade energética em relação aos de origem animal, evidenciando a recomendação nacional vigente de que os alimentos in natura, predominantemente os de origem vegetal, representem a base 
da alimentação ${ }^{17}$. Uma pesquisa realizada com adultos norte-americanos ( $\geq 19$ anos) observou que o aumento da ingestão de fibras contribuiu significativamente para o aumento do escore de qualidade da dieta ${ }^{45}$, mostrando, assim como nos resultados do presente estudo, que este nutriente é um importante marcador de consumo alimentar saudável.

Entre as limitações do estudo deve-se considerar que a aplicação de um único R24h não representa a ingestão habitual dos adolescentes devido à variabilidade do consumo alimentar ${ }^{19}$. Contudo, o R24h é considerado um instrumento adequado para estimar a ingestão média de alimentos e nutrientes quando aplicado em base populacional e de modo a considerar os diferentes dias da semana e meses do ano.

Outra limitação refere-se ao uso da pergunta sobre autoavaliação da qualidade da dieta, um constructo ainda pouco explorado na literatura. Em 2017, Rodrigues et al. ${ }^{46}$ investigaram a aplicabilidade da pergunta "Em sua opinião, a qualidade da sua alimentação é?” para avaliar a qualidade da dieta de adolescentes, e constataram sensibilidade de $28 \%$ e especificidadede $79 \%$ para detectar dietas de boa e má qualidade nutricional, respectivamente. Os autores apontaram limitação no uso da pergunta em adolescentes, dado que alimentos como biscoitos, doces, bebidas adoçadas e fast-food não foram percebidos como parte de uma alimentação de qualidade ruim ${ }^{46}$. Neste estudo foi observada menor ingestão de fibras entre os que avaliaram a qualidade da dieta como ruim, resultado alinhado com o obtido em pesquisa que verificou relação inversa entre ingestão de produtos ultraprocessados e fibra alimentar ${ }^{35}$.

Este estudo traz informações sobre a ingestão de fibra alimentar total e das frações solúvel e insolúvel, e apresenta as fontes alimentares do nutriente segundo a classificação NOVA em uma amostra representativa da população de adolescentes do município de Campinas. Os resultados denotam a ingestão insuficiente de fibras em todos os subgrupos analisados, que os alimentos in natura/minimamente processados forneceram quase $70 \%$ do teor de fibras totais da dieta, e que o consumo de energia, gorduras, açúcar livre e proteína de origem animal diminuiu com o aumento da ingestão do nutriente.

Estes achados reforçam a importância da recomendação de que os alimentos in natura ou minimamente processados ${ }^{17}$ constituam a base da alimentação e assim, possam contribuir para o planejamento de ações que promovam a alimentação saudável e adequada em nível individual e familiar.

\section{Colaboradores}

RCF Meira participou da proposta do artigo, revisão da literatura e redação do texto. CD Capitani, AA Barros Filho e MBA Barros realizaram a revisão crítica do conteúdo intelectual e das análises estatísticas. D Assumpção orientou a proposta do artigo, realizou a análise dos dados e a redação do texto. Todos os autores aprovaram a versão final do manuscrito. 


\section{Agradecimentos}

A Fundação de Amparo à Pesquisa do Estado de São Paulo (FAPESP) pelo financiamento das pesquisas ISACamp 2014-2015 e ISACamp-Nutri 2015-2016.

\section{Referências}

1. Ma Y, Hu M, Zhou L, Ling S, Li Y, Kong B, Huang P. Dietary fiber intake and risks of proximal and distal colon cancers: A meta-analysis. Medicine (Baltimore) 2018; 97(36):e11678.

2. Threapleton DE, Greenwood DC, Evans CEL, Cleghorn CL, Nykjaer C, Woodhead C, Cade JE, Gale CP, Burley VJ. Dietary fibre intake and risk of cardiovascular disease: systematic review and meta-analysis. BMJ 2013; 347:f6879.

3. McRae MP. Dietary Fiber Is Beneficial for the Prevention of Cardiovascular Disease: An Umbrella Review of Meta-analyses. J Chiropr Med 2017; 16(4):289-299.

4. Kim Y, Je Y. Dietary fibre intake and mortality from cardiovascular disease and all cancers: A meta-analysis of prospective cohort studies. Arch Cardiovasc Dis 2016; 109(1):39-54.

5. Bhupathiraju SN, Tobias DK, Malik VS, Pan A, Hruby A, Manson JE, Willett WC, Hu FB. Glycemic index, glycemic load, and risk of type 2 diabetes: results from 3 large US cohorts and an updated meta-analysis. Am J Clin Nutr 2014; 100(1):218-232.

6. Lin Y, Huybrechts I, Vereecken C, Mouratidou T, Valtueña J, Kersting M, González-Gross M, Bolca S, Wärnberg J, Cuenca-García M, Gottrand F, Toti E, Gomez-Martínez S, Grammatikaki E, Labayen I, Moreno LA, Sjöström M, Van Camp J, Roccaldo R, Patterson E, Manios Y, Molnar D, Kafatos A, Widhalm K, De Henauw S. Dietary fiber intake and its association with indicators of adiposity and serum biomarkers in European adolescents: the HELENA study. Eur J Nutr 2015; 54(5):771-782.

7. Albertson AM, Reicks M, Joshi N, Gugger CK. Whole grain consumption trends and associations with body weight measures in the United States: results from the crosssectional National Health and Nutrition Examination Survey 2001-2012. Nutr J 2016; 15:8.

8. Sardinha AN, Canella DS, Martins APB, Claro RM, Levy RB. Dietary sources of fiber intake in Brazil. Appetite 2014; 79(1):134-138.

9. McGill CR, Fulgoni VL, Devareddy L. Ten-Year Trends in Fiber and Whole Grain Intakes and Food Sources for the United States Population: National Health and Nutrition Examination Survey 2001-2010. Nutrients 2015; 7(2):1119-1130.

10. Fayet-Moore F, Cassettari T, Tuck K, McConnell A, Petocz P. Dietary Fibre Intake in Australia. Paper I: Associations with Demographic, Socio-Economic, and Anthropometric Factors. Nutrients 2018; 10(5):599.

11. Instituto Brasileiro de Geografia e Estatística (IBGE). Pesquisa de Orçamentos Familiares 2008-2009: análise do consumo alimentar pessoal no Brasil. Rio de Janeiro: IBGE, 2011.

12. Codex Alimentarius. International Food Standards. Guidelines on Nutrition Labelling: CAC/GL 2-1985 [Internet]. [acessado 2019 fev 25]. Disponível em: http://www.fao.org/fao-who-codexalimentarius/codex-texts/guidelines/en/.

13. Stephen AM, Champ MM-J, Cloran SJ, Fleith M, van Lieshout L, Mejborn H, Burley VJ. Dietary fibre in Europe: current state of knowledge on definitions, sources, recommendations, intakes and relationships to health. Nutr Res Rev 2017; 30(2):149-190. 
14. O'Grady J, O'Connor EM, Shanahan F. Review article: dietary fibre in the era of microbiome science. Aliment Pharmacol Ther 2019; 49(5):506-515.

15. Eswaran S, Muir J, Chey WD. Fiber and functional gastrointestinal disorders. Am J Gastroenterol 2013; 108(5):718-727.

16. Dhingra D, Michael M, Rajput H, Patil RT. Dietary fibre in foods: a review. J Food Sci Technol 2012; 49(3):255-266.

17. Brasil. Ministério da Saúde (MS). Secretaria de Atenção à Saúde. Departamento de Atenção Básica. Guia Alimentar para a População Brasileira. 2a ed. Brasília: MS; 2014.

18. Alves MCGP. Plano de Amostragem do ISACAMP-2014/15 [Internet]. [acessado 2019 fev 25]. Disponível em: https://www.fcm.unicamp.br/fcm/ sites/default/files/2018/page/plano_de_amostragem_ isacamp_2014.15.pdf.

19. Domene SMA. Avaliação do consumo alimentar. In: Taddei JAAC, Lang RMF, Longo-Silva G, Toloni MHA, organizadores. Nutrição em saúde pública. Rio de Janeiro: Rubio; 2011. p. 41-54.

20. Steinfeldt L, Anand J, Murayi T. Food reporting patterns in the USDA Automated Multiple-Pass Method. Procedia Food Sci 2013; 2:145-156.

21. Fisberg RM, Villar BS. Manual de receitas e medidas caseiras para cálculo de inquéritos alimentares: manual elaborado para auxiliar o processamento de inquéritos alimentares. São Paulo: Signus; 2002.

22. Pinheiro ABV, Lacerda EMA, Benzecry EH, Gomes MCS, Costa VM. Tabela para avaliação de consumo alimentar em medidas caseiras. $5^{\mathrm{a}}$ ed. São Paulo: Editora Atheneu; 2004.

23. Araújo MOD, Guerra TMM. Alimentos "Per Capita". Natal: Editora Universitária; 1992.

24. Maia EG, Silva LES, Santos MAS, Barufaldi LA, Silva SU, Claro RM. Padrões alimentares, características sociodemográficas e comportamentais entre adolescentes brasileiros. Rev Bras Epidemiol 2018; 21(Supl. 1):E180009.

25. Forkert ECO, Moraes ACF, Carvalho HB, Manios Y, Widhalm K, González-Gross M, Gutierrez A, Kafatos A, Censi L, Henauw SD, Moreno LA. Skipping breakfast is associated with adiposity markers especially when sleep time is adequate in adolescents. Sci Rep 2019; 9:6380.

26. Organização Pan-Americana da Saúde (OPAS). Plano de Ação para Prevenção da Obesidade em Crianças e Adolescentes. Washington, DC: OPAS; 2014.

27. Carvalho AM, Piovezan LG, Selem SSC, Fisberg RM, Marchioni DML. Validação e calibração de medidas de peso e altura autorreferidas por indivíduos da cidade de São Paulo. Rev Bras Epidemiol 2014; 17(3):735746.

28. Brasil. Ministério da Saúde (MS). Secretaria de Atenção à Saúde. Departamento de Atenção Básica. Orientações para a coleta e análise de dados antropométricos em serviços de saúde: Norma Técnica do Sistema de Vigilância Alimentar e Nutricional - SISVAN. Brasília: MS; 2011.
29. Monteiro CA, Cannon G, Moubarac J-C, Levy RB Louzada MLC, Constante PJ. The UN Decade of Nutrition, the NOVA food classification and the trouble with ultra-processing. Public Health Nutr 2017; 21(1):5-17.

30. World Health Organization (WHO). Diet, nutrition and the prevention of chronic diseases. Technical report series 916. Genebra: WHO; 2003.

31. World Health Organization (WHO). Guideline: Potassium intake for adults and children. Genebra: WHO; 2012.

32. Institute of Medicine. Dietary Reference Intakes for Energy, Carbohydrate, Fiber, Fat, Fatty Acids, Cholesterol, Protein, and Amino Acids [Internet]. Washington, DC: The National Academies Press; 2005 [acessado 2019 fev 25]. Disponível em: https://www.nap.edu/ download/10490.

33. Levy RB, Claro RM, Mondini L, Sichieri R, Monteiro CA. Distribuição regional e socioeconômica da disponibilidade domiciliar de alimentos no Brasil em 20082009. Rev Saude Publica 2012; 46(1):6-15.

34. Martins APB, Levy RB, Claro RM, Moubarac JC, Monteiro CA. Participação crescente de produtos ultraprocessados na dieta brasileira (1987-2009). Rev Saude Publica 2013; 47(4):656-665.

35. Louzada MLC, Martins APB, Canella DS, Baraldi LG, Levy RB, Claro RM, Moubarac J-C, Cannon G, Monteiro CA. Alimentos ultraprocessados e perfil nutricional da dieta no Brasil. Rev Saude Publica 2015; 49:38.

36. Monteiro CA, Moubarac J-C, Levy RB, Canella DS, Louzada MLC, Cannon G. Household availability of ultra-processed foods and obesity in nineteen European countries. Public Health Nutr 2017; 21(1):18-26.

37. Li YO, Komarek AR. Dietary fibre basics: Health, nutrition, analysis, and applications. Food Qual Saf 2017; 1(1):47-59.

38. U.S. Food and Drug Administration. Science Review of Isolated and Synthetic Non-Digestible Carbohydrates [Internet]. [acessado 2019 fev 12]. Disponível em: https://www.fda.gov/Food/LabelingNutrition/ucm 525656.htm

39. Alves MA, Souza AM, Barufaldi LA, Tavares BM, Bloch KV, Vasconcelos FAG. Padrões alimentares de adolescentes brasileiros por regiões geográficas: análise do Estudo de Riscos Cardiovasculares em Adolescentes (ERICA). Cad Saude Publica 2019; 35(6):e00153818.

40. Azeredo CM, Rezende LFM, Canella DS, Claro RM, Castro IRR, Luiz OC, Levy RB. Dietary intake of Brazilian adolescents. Public Health Nutr 2015; 18(7):1215-1224.

41. Barufaldi LA, Abreu GA, Oliveira JS, Santos DF, Fujimori E, Vasconcelos SML, Vasconcelos FAG, Tavares BM. ERICA: prevalência de comportamentos alimentares saudáveis em adolescentes brasileiros. Rev Saude Publica 2016; 50(Supl. 1):6s.

42. Marchioni DML, Gorgulho BM, Teixeira JA, Verly Junior E, Fisberg RM. Prevalência de omissão do café da manhã e seus fatores associados em adolescentes de São Paulo: estudo ISA-Capital. Nutrire 2015; 40(1):10-20. 
43. Coulthard JD, Palla L, Pot GK. Breakfast consumption and nutrient intakes in 4-18-year-olds: UK National Diet and Nutrition Survey Rolling Programme (20082012). Br J Nutr 2017; 118(4):280-290.

44. Carvalho SDL, Barros Filho AA, Barros MBA, Assumpção D. Qualidade da dieta segundo a autoavaliação de adolescentes: resultados do ISACamp-Nutri. Cien Saude Colet 2020; 25(11):4451-4461.

45. McGill CR, Birkett A, Fulgonii VL. Healthy Eating Index-2010 and food groups consumed by US adults who meet or exceed fiber intake recommendations NHANES 2001-2010. Food Nutr Res 2016; 60:29977.

46. Rodrigues PRM, Gonçalves-Silva RMV, Ferreira MG, Pereira RA. Viabilidade do uso de pergunta simplificada na avaliação da qualidade da dieta de adolescentes. Cien Saude Colet 2017; 22(5):1565-1578.

Artigo apresentado em 13/05/2019

Aprovado em 19/05/2020

Versão final apresentada em 21/05/2020

Editores-chefes: Romeu Gomes, Antônio Augusto Moura da Silva 